\title{
Sprechende Zahnmedizin Teil 2: GOÄ-Nr. 60
}

\author{
Mit Ausführungen um die GOÄ-Nr. 4 (Fremdanamnese und Instruktion von Bezugspersonen) ist \\ eine kleine Artikelserie zur Berechnung der sprechenden Zahnmedizin in der DFZ-Januarausgabe \\ gestartet. Gespräche mit ärztlichen oder zahnärztlichen Kollegen sind nicht minder wichtig. \\ Deshalb steht jetzt die Berechnung der GOÄ-Nr. 60 im Fokus, deren Leistungslegende im Voll- \\ text lautet: „Konsiliarische Erörterung zwischen zwei oder mehr liquidationsberechtigten Ärzten, \\ für jeden Arzt".
}

In der DFZ-Januarausgabe wurde mit Blick auf die BEMA-Nrn. 181 und 182 (Konsile bei GKV-Versicherten) auf die immer häufiger erforderliche allgemeinärztliche oder fachärztliche konsiliarische Abstimmung hingewiesen. Hintergrund sind zunehmend multimorbide Patienten und beispielsweise Nebenwirkungen neuer oder vermehrt angewendeter Medikamente (neue orale Koagulanzien, Bisphosphonate).

Ein Konsil ist die Beratung mehrerer Ärzte über einen Krankheitsfall. Ein Konsilium wird abgehalten, wenn in einem Krankheitsfall der Rat von mehreren Ärzten (oftmals unterschiedlicher Fachrichtungen) gehört werden soll. Dabei ist wesentlich, dass zum Konsil der persönliche Kontakt jedes Konsiliarius mit dem Patienten gehört.

\section{Gegenseitiger Austausch}

Bei einer zahnärztlichen Tätigkeit ist das Zusammenfinden und der gegenseitige Austausch in solchen Krankheitsfällen, bei denen sich gemeinsam ein Zahnarzt und beispielsweise ein Hausarzt, ein Internist, ein Mund-, Kiefer- und Gesichtschirurg oder ein Hals-Nasen-Ohren-Arzt beraten, als Konsil anzusehen. Auch das gemeinsame Beraten von zwei oder mehr Ärzten derselben Fachrichtung (also zum Beispiel auch von zwei Zahnärzten, etwa Hauszahnarzt und Kieferorthopäde; Hauszahnarzt und Oralchirurg; Hauszahnarzt und spezialisierter anderer Zahnarzt) über einen Krankheitsfall stellt ein Konsil dar und kann als solches berechnet werden.

Dabei ist ein direkter persönlicher Kontakt zwischen den Ärzten vis-à-vis nicht zwingend nötig, das Konsil kann auch telefonisch oder auf den üblichen anderen Wegen der Telekommunikation erfolgen. Neben der niedergelassenen Praxis können Zahnärzte in der Funktion als Konsiliarius auch in fremden Praxen oder im

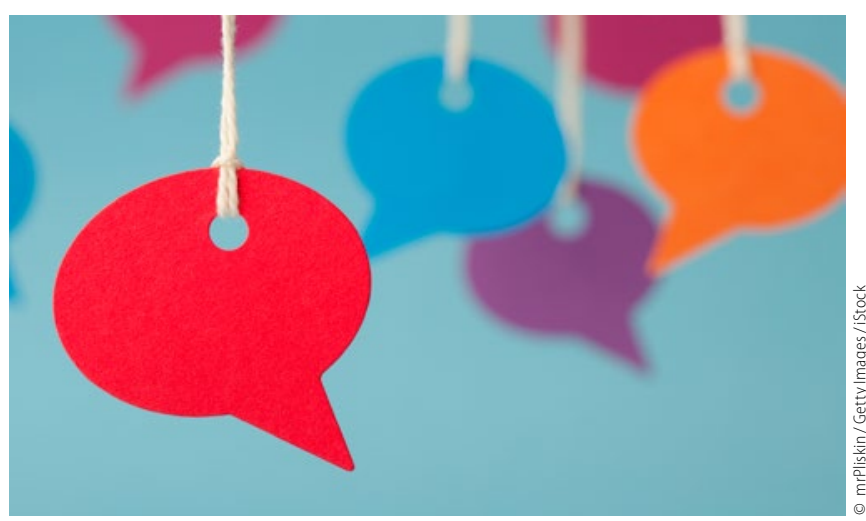

stationären Zusammenhang eines Krankenhauses oder eines Pflegeheimes tätig werden.

\section{Persönlich mit dem Patienten befasst}

Die GOÄ-Nr. 60 kann von jedem liquidationsberechtigten Konsiliarius, der an einem Krankheitsfall beratend tätig ist, berechnet werden. Nach der Abrechnungsbestimmung darf die Leistung nach der GOÄ-Nr. 60 aber nur dann berechnet werden, wenn sich der liquidierende Arzt oder Zahnarzt zuvor oder in unmittelbarem zeitlichem Zusammenhang mit der konsiliarischen Erörterung persönlich mit dem Patienten und dessen Erkrankung befasst hat.

Routinemäßige Zusammenkünfte (beispielsweise Dienstbesprechungen, Teambesprechungen, Patientenübergaben Anästhesist/Operateur) stellen allerdings keine Konsile dar. Wenn die zusammenkommenden (Zahn-)Ärzte Mitglieder derselben Praxisgemeinschaft (bei gleicher oder ähnlicher Fachrichtung) oder Gemeinschaftspraxis oder Krankenhausabteilung sind, so stellt dies im Sinne der GOÄ ebenfalls kein Konsil dar, und die Berechnung der GOÄ-Nr. 60 ist hier nicht möglich. Ebenfalls sind Beratungen mit Nichtärzten (wie Zahntechniker, Physiotherapeuten, Logopäden) nicht nach der GOÄ-Nr. 60 als Konsil berechnungsfähig.

Ist ein Konsil sehr zeitintensiv, so ist nach $₫ 5$ GOÄ die Gebührenhöhe der GOÄ-Nr. 60 steigerungsfähig.

\section{Zuschläge $\mathrm{E}$ bis $\mathrm{H}$ der GOÄ}

Nicht vergessen werden sollte auch, dass der nicht steigerungsfähige Zuschlag E der GOÄ (160 Punkte = 9,33 Euro) für dringend angeforderte und unverzüglich erfolgte Konsile zusätzlich berechnet werden kann. Getrennt davon existieren übrigens auch die GOÄ-Zuschläge nach den Buchstaben F, G und/oder $\mathrm{H}$ der GOÄ für jeweilige zeitliche Besonderheiten.

Die Ausführungen beruhen auf dem Gebührenkommentar von Liebold/Raff/Wissing zu GOZ und BEMA.

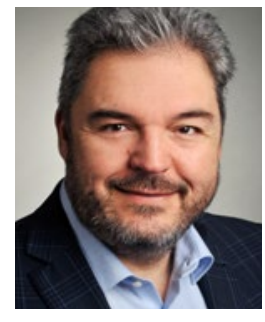

Dr. Dr. Alexander Raff

Mitglied im GOZ-Expertenrat des FVDZ 